\title{
Immunogenicity of nonreplicating recombinant vaccinia expressing HLA-A201 targeted or complete MART-1/Melan-A antigen
}

\author{
Alexander Schütz, ${ }^{1}$ Daniel Oertli, ${ }^{2}$ Walter R. Marti, ${ }^{2}$ Christoph Noppen, ${ }^{3}$ \\ Elisabetta Padovan, ${ }^{3}$ Giulio C. Spagnoli, ${ }^{3}$ Michael Heberer, ${ }^{3}$ and Paul Zajac ${ }^{3}$ \\ ${ }^{1}$ Surgical Department, University Hospital, Regensburg, Germany; ${ }^{2}$ Surgical Department, University Hospital, \\ Basel, Switzerland; ${ }^{3}$ Research Unit of Surgical Department, University Hospital, Basel, Switzerland.
}

The effect on immunogenicity of different tumor T cell epitope formulations was evaluated in vitro using nonreplicating recombinant vaccinia vector expressing two forms of the melanoma - associated MART - 1/Melan-A antigen. The first recombinant virus expressed a minigene encoding a fusion product between an endoplasmic reticulum (ER) -targeting signal and the HLA-A201 binding $27-35$ peptide. The second viral construct encoded the complete MART - 1/Melan-A protein. The capacity of HLA-A201 cells infected with either viral construct to generate and to stimulate MART-1/Melan-A 27-35 specific cytotoxic T-lymphocytes (CTL), was comparatively characterized. The results obtained here with a tumor antigen confirmed the capacity of vaccinia virus-encoded ERminigene to generate a very strong antigenic signal. In cytotoxicity assays, recognition of target cells infected with high amounts of both recombinant viruses with activated specific CTL clones, resulted in similar lytic activity. With regard to calcium mobilization, TCR down-regulation, IFN - $\gamma$ release, and T cell proliferation assays, the targeted epitope elicited 10- to 1000-fold stronger responses. Remarkably, the immunogenic difference between the two formulations, in their respective capacity to generate $C T L$ from naive HLA-A2 peripheral blood mononuclear cells in vitro as measured by tetramer detection, was lower (2- to 3-fold). Recombinant vectors expressing complete antigens have demonstrated their capacity to generate specific responses and such vaccines might take advantage of a broader potential of presentation. However, as demonstrated here for the HLA-A201-restricted MART - 1 / Melan - A immunodominant epitope, nonreplicative vaccinia virus expressing ER - targeted minigenes appear to represent a significantly more immunogenic epitope vaccine formulation. Cancer Gene Therapy (2001) 8, 655-661

Key words: MART-1/Melan-A; minigene; vaccinia virus; immunogenicity.

$\mathrm{T}$ he goal of antitumor specific immunotherapy is to elicit specific cytotoxic T cells (cytotoxic T-lymphocytes or CTL) that recognize and destroy cancer cells expressing the targeted tumor antigen. Design of vaccines should fulfill requirements of tumor cells' biology and CTL activation mechanisms. ${ }^{13,14}$ In nonvirus-related cancers, tumor antigens are self-proteins mutated or with a different pattern of expression compared to normal differentiated tissues. Natural immune responses, against these tumor-associated antigens, are generally weak or absent. Therefore, many groups have evaluated vaccines that could efficiently break this tolerance and induce specific immune responses. Synthetic peptides of HLA - restricted epitopes, derived from tumor-associated antigens, have been used in vitro and in vivo and have indeed demonstrated their capacity to elicit specific responses. 9,19 Modification of peptide sequences also led to the definition of more immunogenic reagents. ${ }^{15,18}$ Recently, HLA-A201 MART-1/Melan-A 27-35 peptide analogues or the corresponding mutated complete antigen

Received May 29, 2001.

Address correspondence and reprint requests to Dr. Paul Zajac, Ph.D., ZLF, Hebelstrasse 20, 4031, Basel, Switzerland. E-mail address: pzajac@uhbs.ch were compared using vaccinia virus expression vectors. ${ }^{23}$ This work showed evidence that analogues of the immunodominant epitope, encoded by minigenes or those processed from a modified complete protein, had a stronger immunogenicity than the natural epitope.

In the present work, we addressed the relative immunogenicity of two recombinant vaccinia viruses (rVV) providing the MART-1/Melan-A 27-35 epitope. The first viral recombinant contains a minigene encoding an endoplasmic reticulum (ER) - targeted peptide (rVV-minigene) as described previously. ${ }^{27}$ The second viral construct expresses the fullgene encoding the natural complete protein ( $\mathrm{rVV}$-fullgene). Both formulations are encoded by replication-incompetent vaccinia virus, enabling an efficient intracellular expression of the genes of interest with no cytopathic effects on the infected cells. These constructs were evaluated for their respective capacity to generate and stimulate in vitro anti-MART 27-35 CTL.

\section{MATERIALS AND METHODS}

Peptide, virus, cell lines, CTL

MART-1/Melan-A 27-35 (AAGIGILTV) and control peptide GP100 280-288 (YLEPGVTA) were purchased 
from Orpegen Therapeutika, Heidelberg, Germany. The construction of a vaccinia virus encoding the ER-targeted MART 27-35 peptide was previously described. ${ }^{27}$ The complete open reading frame of MART-1/Melan-A was PCR-cloned from a patient-derived primary melanoma cell line cDNA. The gene was then transferred into a vaccinia specific shuttle plasmid, derived from pKT1401, enabling insertion in the I4L locus and its expression under the control of a modified early viral promoter (courtesy of Dr. K. Tsung ${ }^{21}$ ).

Virus selection, amplification, and titration were performed on CV1 cell cultures (ATCC CCL70) maintained in DMEM-10\% heat inactivated FCS (Gibco-BRL, Pasley, UK). The final viral genomic sequence of the MART-1/ Melan-A insert was verified. A psoralen-UV inactivation step was performed to obtain nonreplicating working stock virus as described earlier. ${ }^{21,27}$ The resulting UV-inactivated vaccinia virus is able to infect and to transcribe its early promoters more efficiently than replicating virus. However, it does not affect the cell survival nor does it produce any viral progeny.

MART-1/Melan-A 27-35 specific CTL clones were generated in our laboratory from HLA-A2 peripheral blood mononuclear cell (PBMC) cultures stimulated with autologous Epstein-Barr virus transformed B-lymphocytes (EBV-BL) infected with rVV-minigene (priming) or pulsed with the immunodominant nonamer peptide (boosting). $\mathrm{CD} 8(+)$ specific clones were isolated by limiting dilution, tested for their specificity, and maintained in complete medium containing human sera (CM-AB) together with cytokines (RPMI 1640 supplemented with 1 $\mathrm{mM} \mathrm{Na}$-pyruvate, $2 \mathrm{mM}$ L-glutamine, $10 \mathrm{mM}$ Hepes buffer [all from Life-Technology, Pasley, UK], $20 \mu \mathrm{g} / \mathrm{mL}$ Ciproxin [Bayer, Zurich, Switzerland], $7.5 \%$ pooled $\mathrm{AB}$ human serum, $100 \mathrm{U} / \mathrm{mL}$ IL-2, and $20 \mathrm{U} / \mathrm{mL}$ IL-4). Cell culture expansion was performed at monthly intervals by using irradiated allogenic PBMC and PHA $(1.5 \mu \mathrm{g} / \mathrm{mL})$. HLA-A2 positive MART-1/Melan-A negative melanoma Na-8 cells (courtesy of Dr. F. Jotereau, Nantes, France) were used as target/stimulator cells.

\section{Infection of $\mathrm{Na}-8$ with inactivated vaccinia virus}

Na-8 cell monolayers, in 6- or 12-well plates (Becton Dickinson, Franklin Lakes, NJ), were infected with different amounts of inactivated virus, to achieve the indicated multiplicity of infection (MOI) (wild type infection, as negative control, was only performed at the highest multiplicity). This viral solution adsorption was left for 1 hour at $37^{\circ} \mathrm{C}$ with regular gentle agitation before being replaced by normal CM-10\% FCS medium. The infected cells were further incubated for 16-20 hours at $37^{\circ}$ then trypsineharvested, washed, counted, and used in the different tests as further described.

\section{Cytotoxicity assay}

Cytolytic activities were measured using standard 4 hours chromium release assays. CTL were resuspended in CM$20 \%$ FCS and distributed in a 96-well plate at different densities. Infected or pulsed target cells were labeled with
$\mathrm{Na}^{51} \mathrm{Cr}$ for 1 hour at $37^{\circ}$, washed, counted, and finally added onto the effector CTL. After 4 hours co-incubation, supernatants were harvested and released radioactivity was measured in a gamma-counter. Data are reported here as percentage of specific lysis of target cells (percentage lysis on $\mathrm{rVV}$ infected targets - percentage lysis on VV-wild type infected targets). The optimal lytic activity measurable in each condition is defined as the specific lysis observed with saturating concentration of exogenous pulsing $(10 \mu \mathrm{M}$ for 2 hours) with synthetic $27-35$ peptide.

\section{CD3-TCR down-regulation}

$1 \times 10^{5}$ effector CTL were mixed with $2 \times 10^{5}$ infected stimulators in $\mathrm{CM}-10 \%$ FCS for $4-6$ hours at $37^{\circ}$. The cells were then washed, resuspended in ice-cold PBS $/ 0.5 \mathrm{M}$ EDTA, and stained with CD3 and CD8 monoclonal labeled antibodies (Becton Dickinson, Mountain View, CA). Data are reported as percentage of decrease, on antigenic stimulation, of CD3 mean fluorescence intensity using control stimulated CTL as baseline of maximum expression of the CD3 molecule and CD3 negative cells as minimum expression.

\section{Calcium flux}

Effector CTL cells were preincubated with $10 \mu \mathrm{M}$ Indo-1 AM (Sigma, Buchs, Switzerland) for 45 minutes and then washed three times in PBS and resuspended in CM without phenol red. Antigen presenting cells (APC) were washed and resuspended in the same medium. Both cell types were then kept at $37^{\circ}$ until use. For each condition, infected/ pulsed APC were added to the CTL, centrifuged 1 minute at $1000 \mathrm{rpm}$, incubated for 1 minute at $37^{\circ}$, then rapidly resuspended for acquisition. Using FACS technology, cells were analyzed for 5 minutes. Calcium-mobilized cells were characterized by an increased $405 \mathrm{~nm} / 525 \mathrm{~nm}$ ratio compared to negative control stimulated cells. Results are represented as percentage of cells above the control value.

\section{Cytokine release/ $T$ cell proliferation}

After irradiation, infected stimulator cells were washed, resuspended in $\mathrm{CM}-\mathrm{AB}$, and mixed with the effector $\mathrm{CTL}$ in a 96 -well plate (flat bottom - Becton Dickinson) at a ratio of two APC for one responding cell (100000 CTL per condition). Each condition was set in triplicate and incubated at $37^{\circ}$. After 24 hours, half of the supernatant was harvested and IFN $-\gamma$ was measured by ELISA (CLB, Amsterdam, Netherlands). After 48 hours, H3 -thymidine solution was added to the cultures for 15 hours. Cells were then collected on filters and the incorporated thymidine was measured in a liquid scintillation counter. Data, resulting from triplicate average of incorporated cpm, are displayed as ratio between each sample and the VV-wt negative control ( stimulation index).

MART-1/Melan-A 27-35 CTL generation from HLA-A2 PBMC

Ficoll-purified PBMC from HLA-A2 healthy donors were seeded onto culture plates in $\mathrm{CM}-7.5 \% \mathrm{AB}$. Autologous 
overnight-infected or peptide-pulsed EBV-BL were irradiated, washed, resuspended in $\mathrm{CM}-7.5 \% \mathrm{AB}$ and added to the PBMC. After 1 week, half of the culture medium was replaced with fresh $\mathrm{CM}-\mathrm{AB}$ containing $20 \mathrm{U} / \mathrm{mL}$ IL-2 and $0.4 \mathrm{U} / \mathrm{mL} \mathrm{IL}-4$. On day 10 , cultures were restimulated with pulsed or infected autologous irradiated EBV-BL. One week later, the population of CD8 + cells bearing the specific TCR for MART 27-35 epitope was assessed by tetramer staining and flow cytometry detection.

\section{RESULTS}

To evaluate the specific immunogenicity of both antigenic forms expressed by recombinant vaccinia, early, intermediate, and late events of T-lymphocytes activation were measured by dedicated functional assays. These cellular responses are not only characterized by their kinetics but also by the different densities of presented antigen required to trigger these responses.

\section{Antigen presentation kinetics in nonreplicating $V V$}

After infection, the time required for each viral construct to reach a stable MART-1/Melan-A 27-35 epitope surface presentation differs depending on the delay required to achieve all the necessary steps of expression, processing into peptides, TAP - dependent transport, and HLA presentation at the cell surface. The targeted epitope is designed to bypass two of these steps, namely cytosolic processing and TAP transport. Therefore, initial experiments established the respective duration of infection required for each recombinant virus to reach a stabilized level of presentation. This

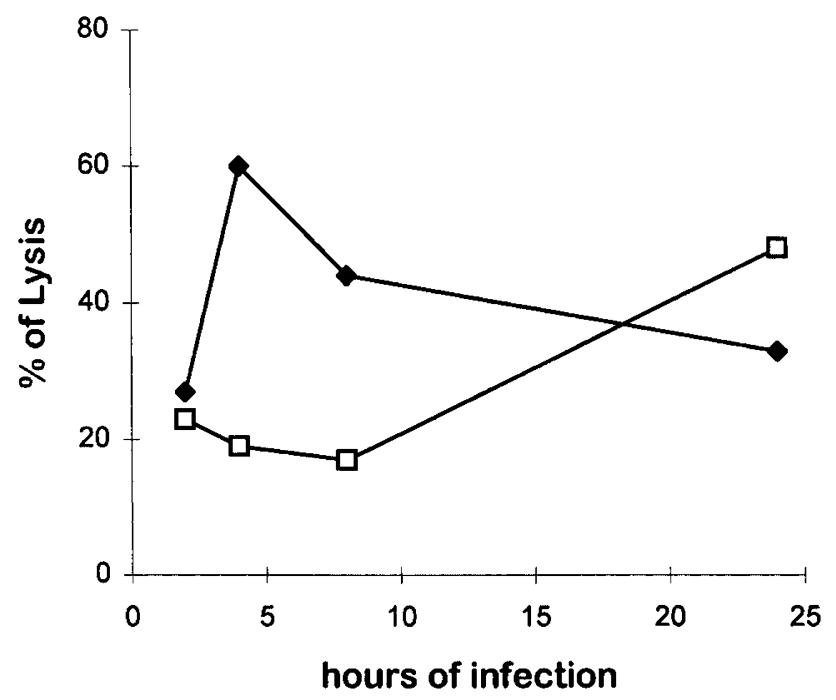

Figure 1. Kinetics of infected target cell sensitization to CTL cytotoxicity. HLA-A2 positive Na-8 cells were infected (10 MOI) with UV-inactivated rVV-minigene $(\diamond)$ or rVV-fullgene $(\square)$ at different times $(2,4,8$, and 24 hours) before the addition of a cytolytic CTL clone. For graphic simplicity, only the results of the highest $C T L$ density $(E T$ ratio $=8$, triplicate) are displayed here as representative of the complete ET ratio pattern. Standard deviations, below $10 \%$ of reported values, are not represented.
A

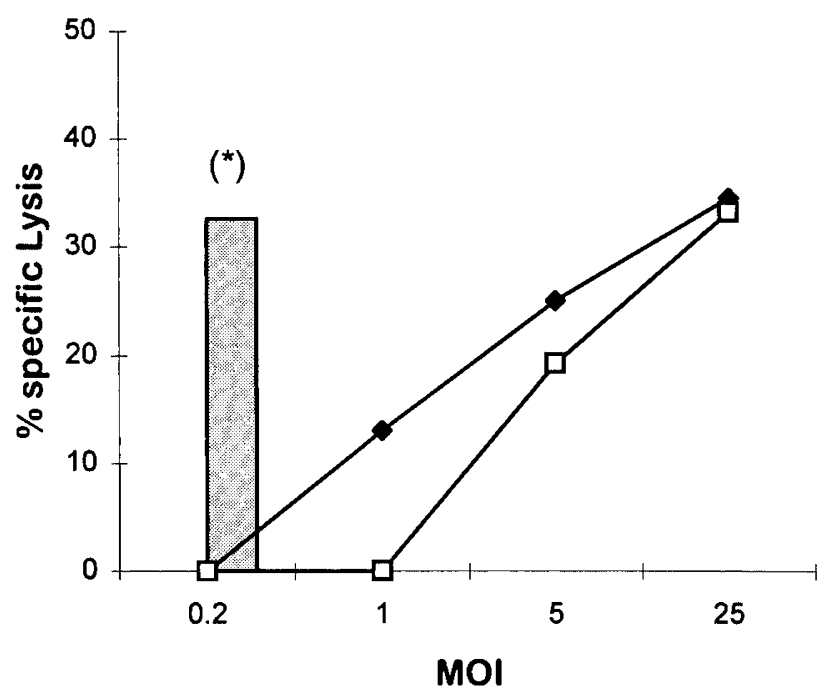

B

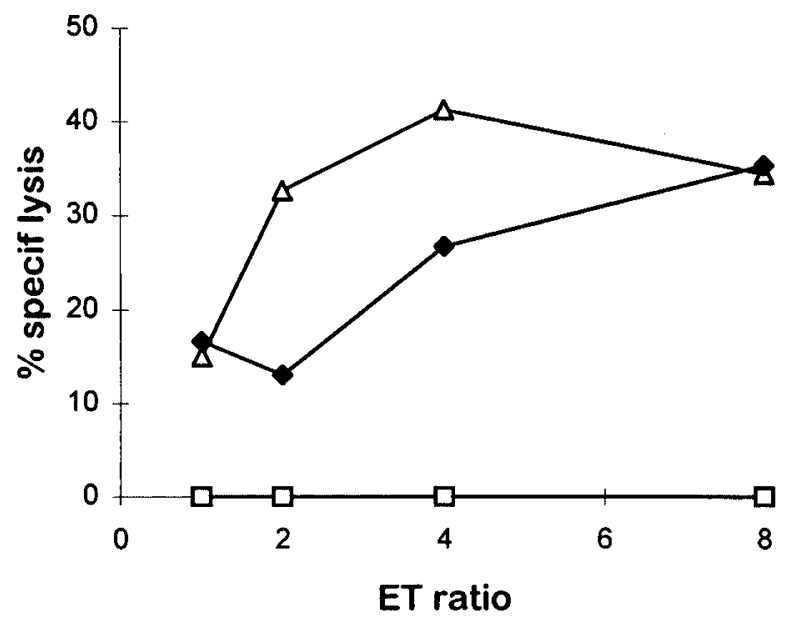

Figure 2. Specific CTL cytotoxic activity on rVV MART - 1/Melan - A 27-35-infected cells. A: Target cell specific lysis obtained with rVVminigene $(\triangleleft)$ or $\mathrm{rVV}$-fullgene $(\square)$ at different MOI (ET ratio=2). Optimal response $\left({ }^{*}\right)$ in these conditions is represented by the activity on similar noninfected cells pulsed with $10 \mu \mathrm{M}$ of MART 2735 peptide. B: Specific cytotoxic activity at different ET ratios observed on infected cells, with rVV-mg and rVV-fg (same label as in panel $\mathbf{A}$ ) in limited infection conditions (one nonreplicationcompetent viral unit), and control pulsed cells $(\triangle)$. Standard deviations, below $10 \%$ of reported values, are not represented.

stable presentation being more suitable for the following quantitative comparisons.

Infected target cells recognition by specific CTL was measured at different times following infection with rVV (10 MOI). Optimal recognition of target cells on infection with ER-minigene rVV (Fig 1) was obtained within a few hours following infection (4 hours). However, optimal 
recognition of cells infected by the rVV-fullgene was found to require longer incubation times, up to 24 hours, to reach similar levels of recognition, consistent with previous reports about UV-inactivated vaccinia expression pattern. ${ }^{11,16}$ Based on these data, overnight infection (16-20 hours) was used throughout the study to allow a stabilized and effective antigen presentation following infection with either rVV.

\section{Target cell sensitization to cytotoxic response}

The cytotoxic activity of a specific CTL clone against target cells infected with different doses of either rVV was evaluated (Fig 2A) and showed a dose-dependent specific lytic response. High-dose infections (5-25 MOI), with both recombinant virus, induced cytolytic recognition reaching levels (from $19 \%$ to $35 \%$ ) similar to the one observed $(33 \%)$ with $10 \mu \mathrm{M}$ exogenous peptide pulsing that is defined to be the optimal recognition reachable in the test. However, with a single UV-inactivated viral unit per cell ( 1 MOI) of rVV-fullgene, no specific killing could be observed (Fig 2B). Remarkably, with the same limited infection conditions, the rVV-minigene still induced a significant lysis, reaching optimal value $(35 \%)$ at an ET ratio of 8 . Thus, regarding cytotoxic sensitization of target cells, high doses of the fullgene MART-1/Melan-A viral construct (at least $10 \mathrm{MOI}$ ) were required to reach the antigenic threshold resulting in cytolytic response. However, even at low doses, the targeted epitope-expressing virus was able to provide a high level of antigenic signal.

\section{TCR down-regulation and calcium flux}

The immediate impact of antigenic recognition by the $T$ cell is proportionally reflected by early cellular events such as cytoplasmic calcium mobilization and TCR endocytosis. The data of calcium flux events (Fig 3A) in the CTL population are represented (detailed in Materials and Methods) for a ratio of one CTL for two APC $(\mathrm{ET}=0.5)$. In these conditions, even APC infected with the highest dose of rVV-fullgene induced $\mathrm{Ca}^{2+}$ in less than $10 \%$ of the CTL population. However, rVV-minigene-infected APC (5-25 MOI) could generate a strong calcium flux in $35-$ $40 \%$ of the CTL. In this test, the effect of the minigeneexpressing virus, at the infectious dose of 1 MOI, appeared to be able to generate a stronger signal than $25 \mathrm{MOI}$ of rVV-fullgene.

Similarly, in TCR down-regulation assay (Fig 3B), conditions using fullgene viral construct infections led to a modest decrease of CD3 molecules from cell surface; $20 \%$ decrease with strongest infection (25 MOI) of rVVfullgene. However, the minigene virus at doses varying from 1 to $25 \mathrm{MOI}$, was able to induce a significant decrease of CD3 labeling ranging between $54 \%$ and $61 \%$. As no increase of this effect could be detected with doses above 1 MOI, one could assume that the maximum stimulation of the
A

C

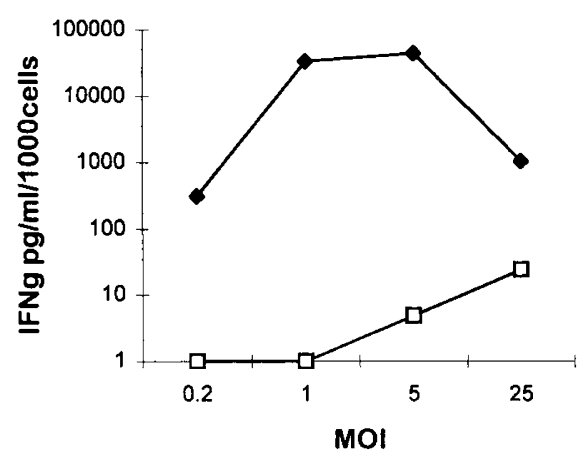

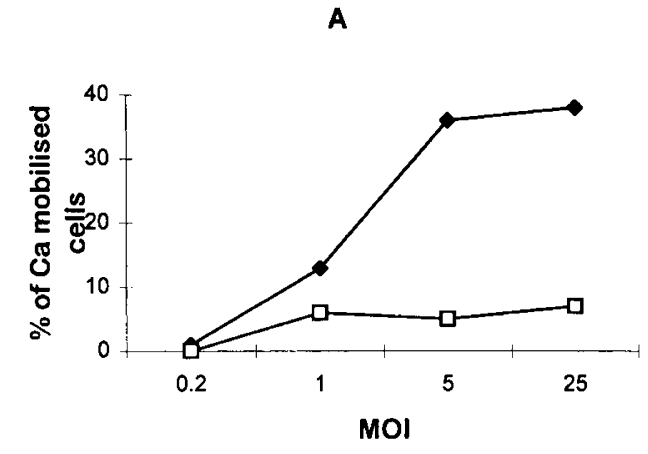

B

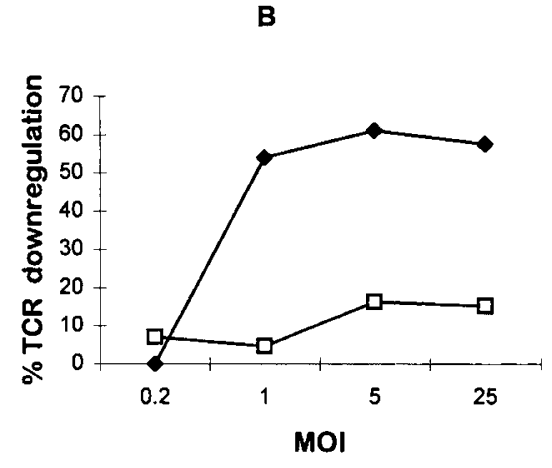

D

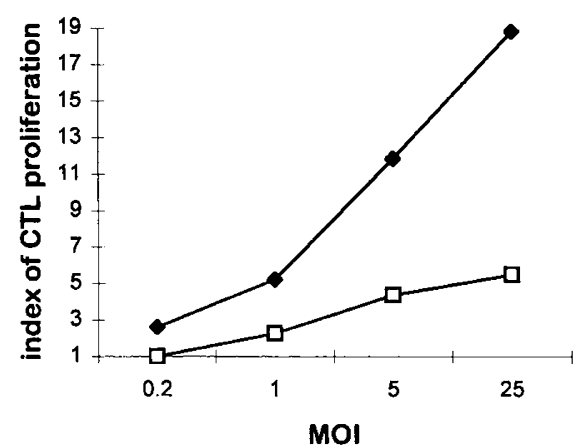

Figure 3. Characterization of dose-dependent rVV-minigene $(\diamond)$ and $r V V$ fullgene $(\square)$ infection's effects on MART-1/Melan-A 27-35 CTL biological responses. HLA - A2 Na- 8 cells were infected at various $\mathrm{MOI}(0.2,1,5$, and 25) and used to stimulate specific CTL (two stimulators for one effector) at time $T=0$. Data obtained with the $\mathrm{VV}$-wild type infected stimulator cells were used as baseline. A: Intracellular calcium mobilization is reported here as percentage of CTL showing an increase calcium signal during the first 5 minutes of contact. B: TCR down-regulation of CD3 molecules from CTL surface, 5 hours after addition of the APC. C: IFN $-\gamma$ release from CTL in culture was measured by ELISA ( $T=24$ hours) in triplicates and reported here as concentration released per 1000 CTL. D: Activation of CTL proliferation was evaluated by 15 hours thymidine incorporation after 2 days of culture. tion after 2 days of culture.

$$
\text { (n) }
$$


TCR as measured by CD3 down-regulation, was already reached at this dose of virus.

\section{Induction of cytokine release}

Besides the early cellular events described above, the induced responses of IFN $-\gamma$ released, following fullgene or minigene viruses stimulation, were strikingly different (Fig 3C). Indeed, a strong cytokine release level (up to $43000 \mathrm{pg} / \mathrm{mL} / 1000$ responders) was reached with the different $\mathrm{APC} / \mathrm{rVV}-\mathrm{mg}$ ratio of $1-25$. Alternatively, only a very limited response (not exceeding $10 \mathrm{pg} / \mathrm{mL} / 1000 \mathrm{CTL}$ ) was observed with the highest dose of rVV-fullgene. Thus, as evaluated by this intermediate cellular event, the rVVfullgene-driven presentation did not appear to reach the threshold of activation required to generate a significant cytokine release.

\section{Induction of $T$ cell proliferation}

The capacity to induce proliferation of activated specific $\mathrm{T}$ cells in vitro is a key feature of the immunogenicity of an antigenic reagent. In this experiment, lymphocyte proliferation, as evaluated by de novo DNA synthesis, was effectively induced by the rVV-minigene (1-25 MOI) between 5 - and 20 -fold compared to the control stimulated cells (Fig 3D). However, the stimulatory capacity of fullgene recombinant infected cells seems to be significantly more limited; the highest doses of virus only resulted in a 2 to 3 -fold increase compared to the control.

\section{MART 27-35 CTL priming capacity from healthy donors'} PBMC

Generation of specific CTL is the ultimate goal of antigen specific immunotherapy. It also possibly represents the best evaluation of an immunogenic compound within a specific prime-boost strategy. The capacity of both recombinant virus to elicit MART-1/Melan-A 27-35 CTL was compared using optimal virus doses as defined above on activated CTL. As displayed in Table 1, two different experimental settings were used. In the first test (A), naive HLA-A2 PBMC were primed with virus-infected autologous APC and restimulated once with synthetic peptide. In

Table 1. Comparative Effect of rVV-Minigene and rVV-Fullgene on MART-1/Melan-A 27-35 CTL Generation

\begin{tabular}{lllcc}
\hline & & & $\begin{array}{c}\text { Tetramer+cells } \\
\text { (as percentage } \\
\text { of CD8+) }\end{array}$ & $\begin{array}{c}\text { Factor } \\
(\mathrm{mg} / \mathrm{fg})\end{array}$ \\
\hline A & V V & Boost & $3.1 \%$ & 3 \\
& VV-fulliningene & Pep & $1.02 \%$ & \\
B & Pep & VV-minigene & $0.43 \%$ & 2 \\
& & VV-fullgene & $0.22 \%$ & \\
\hline
\end{tabular}

PBMC from HLA-A2 healthy donors were primed with infected (A: $20 \mathrm{MOI}$ each virus) or pulsed (B: $20 \mu \mathrm{g} / \mathrm{mL}$ ) autologous irradiated EBV - BL. On day 10, an antigenic boost was operated with peptideloaded (A: $20 \mu \mathrm{g} / \mathrm{mL}$ final) or virus-infected (B: $10 \mathrm{MOI}$ ) autologous EBV-BL. Specific tetramer staining was assessed 7 days after the last stimulation. Amounts of positively stained cells were expressed as percentages of CD8 $(+)$ cells. the second experiment (B), we measured the vaccines' stimulation effect on peptide presensitized PBMC population. Surprisingly, compared to the previous functional characterizations, the calculated ratios between rVV-minigene and rVV-fullgene immunogenicity are here limited to 2 - to 3 -fold in favor of the minigene construct. Both experimental approaches also seem to demonstrate that the stimulation effect of both reagents are relatively similar on a completely naive (A) or peptide-primed (B) PBMC population.

\section{DISCUSSION}

In this study we used MART-1/Melan-A 27-35 specific CTL clones and resting HLA-A201 + PBMC populations to evaluate the relative immunogenicity of two vaccinia constructs expressing either the ER-targeted 27-35 peptide or the cytoplasmic complete protein. Functional assays, addressing different phases of the $\mathrm{T}$ cell activation, were performed with infected HLA-A2+ cells as APC. Different CTL biological responses to antigenic stimulation are characterized by different thresholds of activation ${ }^{7}$ specific for each CTL clone but, most importantly, depending on the level of T cell receptor occupancy ${ }^{22}$ and on the dissociation rate ${ }^{8}$ of the MHC-peptide complex. Although no direct and formal biochemical quantification was performed in this work, our bio-functional tests seem to correlate with the fact also described by others, ${ }^{1}$ that the ER-minigene viral constructs produce and result in the presentation of a greater amount of antigenic epitope compared to the rVV-fullgene. This property results in an enhanced capacity to rapidly (at low dose) reach the activation thresholds of the different specific CTL functions, such as calcium mobilization, TCR down regulation, and cytokine release. Of note, no apparent saturation of the proliferative response could be seen within the conditions of the assay.

This advantage for the ER-minigene virus, compared to the rVV-fullgene, was also confirmed by the results of CTL induction but, in this case, the difference was less remarkable (2- to 3 -fold). This limited difference might be in line with the previous findings that vaccinia-driven over-expression of ER-targeted epitopes enhances the quantitative presentation of specific peptide ${ }^{1}$ but does not always improve the final immunogenicity of an immunodominant epitope. ${ }^{4,6}$

To better understand the relative immunogenicity measured in our tests, the different factors playing a role in this complex system should also be considered. Both antigens are produced within infected cells' cytoplasm, which is optimal for the HLA class-I presentation pathway. The ERminigene design avoids the two limiting steps of cytoplasmic processing and transport. ${ }^{2,27}$ Whereas, the complete antigen is following the complex process of cleavage into peptides by the proteasomal complex, ${ }^{12}$ TAP-dependent transport into the ER where the binding to the appropriate HLA molecule takes place. ${ }^{10}$ Combining with the relative affinity to the different proteins involved in the process, the complete pathway results in nonlinear presentation between peptides of the same protein. ${ }^{1}$ 
Furthermore, the effect of UV inactivation on this viral vector should also be considered. The transcription capacities of the ER-minigene and the fullgene are equivalent and directly proportional to the viral MOI. However, psoralenUV inactivation induces randomized DNA cross-linking. Thus, in similar conditions, the probability of inactivation events of different genes is proportional to their sizes. It is, however, difficult to really estimate the final impact in this case where the fullgene ( $354 \mathrm{bp}$ ) is 4.5 -fold longer than the ER-minigene ( $81 \mathrm{bp}$ ), but where a cross-linking event after the 35th codon might still allow the production of a polypeptide containing the 27-35 epitope. Finally, the immunogenicity of the vector proteins may also interfere. Indeed, the viral proteins and their derived epitopes can also establish some level of immunodominance within the infected cells and this competition may interfere more with the presentation of a processing and transport-dependent epitope (rVV-fullgene) compared to an independent one (rVV-ER-minigene). ${ }^{4,26}$

The use of peptide/minigenes derived from tumorassociated antigen sequences, although efficient in eliciting specific response, sets a limited frame for clinical application. HLA-based selection of patients and phenomenon of tumor escapes from specific (but also more limited) immune response, should be considered as drawback from immunotherapy mediated by a single antigen or restriction element. Many efforts are made to use multiepitopes or complete tumor-associated antigens as immunogenic material and have demonstrated various success in vivo. ${ }^{5,20}$ Indeed, the few HLA-characterized peptides derived from tumor antigens might not represent the entire class-I and class-II presented epitopes profile. This potent diversity is underlined by the demonstrated capacity of the same antigen to generate differently restricted $\mathrm{CTL}^{3,24,25}$ and by the observed capacity of patients with similar HLA haplotype, to respond differently to the same antigen. ${ }^{17}$ Therefore, to design immunogenic reagents, one should carefully evaluate the characteristics and potentials of each antigen to define formulations with more efficient antitumor activity.

Within this frame, our work with psoralen UV-treated vaccinia vector with MART-1/Melan-A immunogenicity in HLA-A201 context, clearly confirmed the notion that targeted minigenes encoding for immunogenic epitopes represent one of the most effective formulation to generate epitope specific immune responses. In addition, generation of specific CTL found in our current clinical study (manuscript in preparation), seems to support the effectiveness of such rVV-ER-minigene vaccines in vivo.

\section{REFERENCES}

1. Anton LC, Yewdell JW, Bennink JR. MHC class I-associated peptides produced from endogenous gene products with vastly different efficiencies. J Immunol. 1997;158:2535-2542.

2. Bacik I, Cox JH, Anderson R, et al. TAP (transporter associated with antigen processing) -independent presentation of endogenously synthesized peptides is enhanced by endoplasmic reticulum insertion sequences located at the amino- but not carboxyl-terminus of the peptide. $J$ Immunol. 1994;152:381-387.

3. Chaux P, Luiten R, Demotte N, et al. Identification of five MAGE-A1 epitopes recognized by cytolytic T lymphocytes obtained by in vitro stimulation with dendritic cells transduced with MAGE-A1. J Immunol. 1999;163:2928-2936.

4. Chen W, Anton LC, Bennink JR, et al. Dissecting the multifactorial causes of immunodominance in class Irestricted $\mathrm{T}$ cell responses to viruses. Immunity. 2000;12: 83-93.

5. Conry RM, Khazaeli MB, Saleh MN, et al. Phase I trial of a recombinant vaccinia virus encoding carcinoembryonic antigen in metastatic adenocarcinoma: comparison of intradermal versus subcutaneous administration. Clin Cancer Res. 1999;5:2330-2337.

6. Deng Y, Yewdell JW, Eisenlohr LC, et al. MHC affinity, peptide liberation, $\mathrm{T}$ cell repertoire, and immunodominance all contribute to the paucity of MHC class I-restricted peptides recognized by antiviral CTL. J Immunol. 1997;158: $1507-1515$.

7. Fonteneau JF, Le Drean E, Le Guiner S, et al. Heterogeneity of biologic responses of melanoma-specific CTL. J Immunol. 1997; 159:2831-2839.

8. Hudrisier D, Kessler B, Valitutti S, et al. The efficiency of antigen recognition by CD8 + CTL clones is determined by the frequency of serial TCR engagement. $J$ Immunol. 1998;161: $553-562$.

9. Jaeger E, Bernhard H, Romero P, et al. Generation of cytotoxic $\mathrm{T}$-cell responses with synthetic melanoma-associated peptides in vivo: implications for tumor vaccines with melanomaassociated antigens. Int J Cancer. 1996;66:162-169.

10. Lehner PJ, Cresswell P. Processing and delivery of peptides presented by MHC class I molecules. Curr Opin Immunol. 1996;8:59-67.

11. Meko JB, Yim JH, Tsung K, et al. High cytokine production and effective antitumor activity of a recombinant vaccinia virus encoding murine interleukin 12. Cancer Res. 1995;55: 4765-4770.

12. Morel S, Levy F, Burlet-Schiltz O, et al. Processing of some antigens by the standard proteasome but not by the immunoproteasome results in poor presentation by dendritic cells. Immunity. 2000;12:107-117.

13. Offringa R, van der Burg SH, Ossendorp F, et al. Design and evaluation of antigen-specific vaccination strategies against cancer. Curr Opin Immunol. 2000;12:576-582.

14. Pardoll DM. Therapeutic vaccination for cancer. Clin Immunol. 2000;95:44-62.

15. Parkhurst MR, Salgaller ML, Southwood S, et al. Improved induction of melanoma-reactive CTL with peptides from the melanoma antigen gp100 modified at HLA-A*0201-binding residues. J Immunol. 1996;157:2539-2548.

16. Puhlmann M, Brown CK, Gnant M, et al. Vaccinia as a vector for tumor-directed gene therapy: biodistribution of a thymidine kinase-deleted mutant. Cancer Gene Ther. 2000;7:66-73.

17. Reynolds SR, Celis E, Sette A, et al. HLA-independent heterogeneity of CD8 + T cell responses to MAGE-3, MelanA/MART-1, gp100, tyrosinase, MC1R, and TRP-2 in vaccine-treated melanoma patients. J Immunol. 1998;161: 6970-6976.

18. Romero P, Gervois N, Schneider J, et al. Cytolytic T lymphocyte recognition of the immunodominant HLAA*0201-restricted Melan-A/MART-1 antigenic peptide in melanoma. J Immunol. 1997;159:2366-2374.

19. Spagnoli GC, Schaefer C, Willimann TE, et al. Peptidespecific CTL in tumor infiltrating lymphocytes from metastatic melanomas expressing MART-1/Melan-A, gp100 and Tyr- 
osinase genes: a study in an unselected group of HLA-A2.1 positive patients. Int J Cancer. 1995;64:309-315.

20. Tsang KY, Zhu M, Nieroda CA, et al. Phenotypic stability of a cytotoxic $\mathrm{T}$-cell line directed against an immunodominant epitope of human carcinoembryonic antigen. Clin Cancer Res. 1997;3:2439-2449.

21. Tsung K, Yim JH, Marti W, et al. Gene expression and cytopathic effect of vaccinia virus inactivated by psoralen and long-wave UV light. J Virol. 1996;70:165-171.

22. Valitutti S, Muller S, Dessing M, et al. Different responses are elicited in cytotoxic $\mathrm{T}$ lymphocytes by different levels of $\mathrm{T}$ cell receptor occupancy. J Exp Med. 1996;183:1917-1921.

23. Valmori D, Levy F, Miconnet I, et al. Induction of potent antitumor CTL responses by recombinant vaccinia encoding a melan-A peptide analogue. J Immunol. 2000;164: $1125-1131$.
24. Yang S, Kittlesen D, Slingluff CLJ, et al. Dendritic cells infected with a vaccinia vector carrying the human gp100 gene simultaneously present multiple specificities and elicit highaffinity $\mathrm{T}$ cells reactive to multiple epitopes and restricted by HLA-A2 and - A3. J Immunol. 2000;164:4204-4211.

25. Yee C, Savage PA, Lee PP, et al. Isolation of high avidity melanoma-reactive CTL from heterogeneous populations using peptide-MHC tetramers. J Immunol. 1999;162:2227-2234.

26. Yewdell J, Anton LC, Bacik I, et al. Generating MHC class I ligands from viral gene products. Immunol Rev. 1999;172:97108.

27. Zajac P, Oertli D, Spagnoli GC, et al. Generation of tumoricidal cytotoxic $\mathrm{T}$ lymphocytes from healthy donors after in vitro stimulation with a replication-incompetent vaccinia virus encoding MART-1/Melan-A 27-35 epitope. Int J Cancer. 1997;71:491-496. 